Documentation et bibliothèques

DOCUMENTATION BIBLIOTHEQUES

\title{
La bibliothèque David M. Stewart
}

La collection de livres anciens du chevalier de Johnstone

The David M. Stewart Library

Colección de libros antiguos del Caballero de Johnstone

\section{Normand Trudel}

Volume 50, numéro 4, octobre-décembre 2004

URI : https://id.erudit.org/iderudit/1030057ar

DOI : https://doi.org/10.7202/1030057ar

Aller au sommaire du numéro

\section{Éditeur(s)}

Association pour l'avancement des sciences et des techniques de la documentation (ASTED)

\section{ISSN}

0315-2340 (imprimé)

2291-8949 (numérique)

\section{Découvrir la revue}

\section{Citer cet article}

Trudel, N. (2004). La bibliothèque David M. Stewart : la collection de livres anciens du chevalier de Johnstone. Documentation et bibliothèques, 50(4), 285-294. https://doi.org/10.7202/1030057ar

\section{Résumé de l'article}

Alors que le Musée Stewart au Fort de l'île Sainte-Hélène s'apprête à fêter ses 50 ans (1955-2005) en rendant hommage à son illustre fondateur, le moment nous est apparu bien choisi pour procéder à un travail de recherche mettant en valeur la bibliothèque David $M$. Stewart. Réalisation particulièrement chère au coeur du grand mécène canadien et à laquelle il a accordé tous ses soins, cette bibliothèque de musée n'a jusqu'à présent jamais fait l'objet d'étude. Nous souhaitons suppléer à ce manque en proposant une première étude exploratoire de la collection de livres anciens du chevalier de Johnstone, qui en constitue le coeur et l'âme.

D'autre part, puisqu'un projet d'inventaire systématique des collections de livres anciens en sol québécois a été récemment initié par la Bibliothèque nationale du Québec (BNQ) et l'Association québécoise de l'imprimé (AQÉI), nous espérons qu'en dressant un portrait de cette riche collection, nous saurons faire oeuvre utile en présentant aux chercheurs ce témoignage d'une des nombreuses contributions de David M. Stewart à l'enrichissement culturel de son pays.

Après une courte présentation de l'homme et de sa philosophie, nous aborderons l'origine et le développement de cette collection particulière qui se veut une reconstitution typique de la bibliothèque d'un gentilhomme de la Nouvelle-France au XVIII ${ }^{\mathrm{e}}$ siècle. Une analyse statistique et un survol des titres nous permettront de voir dans quelle mesure David M. Stewart a réussi à atteindre la mission qu'il s'était donnée.
Tous droits réservés @ $\subseteq$ Association pour l'avancement des sciences et des techniques de la documentation (ASTED), 2004
Ce document est protégé par la loi sur le droit d'auteur. L’utilisation des services d'Érudit (y compris la reproduction) est assujettie à sa politique d'utilisation que vous pouvez consulter en ligne.

https://apropos.erudit.org/fr/usagers/politique-dutilisation/ 


\section{La bibliothèque David M. Stewart ${ }^{1}$ La collection de livres anciens du chevalier de Johnstone}

\author{
NORMAND TRUDEL \\ Archiviste des collections \\ ntrudel@stewart-museum.org
}

\author{
Ab imo pectore \\ (Du fond du cour) \\ Ex-libris de David M. Stewart
}

\section{RÉSUMÉ | ABSTRACTS | RESUMEN}

Alors que le Musée Stewart au Fort de lîle Sainte-Hélène sapprête à fêter ses 50 ans (1955-2005) en rendant hommage à son illustre fondateur, le moment nous est apparu bien choisi pour procéder à un travail de recherche mettant en valeur la bibliothèque David M. Stewart. Réalisation particulièrement chère au cœur du grand mécène canadien et à laquelle il a accordé tous ses soins, cette bibliothèque de musée n'a jusquà présent jamais fait l'objet d'étude. Nous souhaitons suppléer à ce manque en proposant une première étude exploratoire de la collection de livres anciens du chevalier de Johnstone, qui en constitue le cour et liàme.

D'autre part, puisqu'un projet d'inventaire systématique ${ }^{2}$ des collections de livres anciens en sol québécois a été récemment initié par la Bibliothèque nationale du Québec (BNQ) et l'Association québécoise de l'imprimé (AQÉI), nous espérons qu'en dressant un portrait de cette riche collection, nous saurons faire œuvre utile en présentant aux chercheurs ce témoignage d'une des nombreuses contributions de David M. Stewart à l'enrichissement culturel de son pays.

Après une courte présentation de l'homme et de sa philosophie, nous aborderons l'origine et le développement de cette collection particulière qui se veut une reconstitution typique de la bibliothèque d'un gentilhomme de la Nouvelle-France au XVIIre siècle. Une analyse statistique et un survol des titres nous permettront de voir dans quelle mesure David M. Stewart a réussi à atteindre la mission qu'il sẻtait donnée.

\section{The David M. Stewart Library ${ }^{3}$}

The Stewart Museum at the Fort of Île Sainte-Hélène will soon celebrate a half-century of existence (1955-2005) and will pay tribute to its illustrious founder. We thought the time was appropriate to highlight the David. M. Stewart Library. The Library was of special interest to this great Canadian benefactor, who was personally involved in its development. This Library has never been the subject of any research and we wanted to fill a

1. Cet article est un condensé d'un travail de recherche effectué dans le cadre de la maitrise en sciences de l'information à l'Université de Montréal et dirigé par Marcel Lajeunesse.

2. Le projet, présenté au colloque de l'ACFAS du 16 au 18 mai 2004, est au stade embryonnaire.

3. This article is an abridged version of a research paper submitted in partial fulfilment of a Master of Information Sciences degree at the Université de Montréal under the supervision of Marcel Lajeunesse.

4. This project, presented at the ACFAS conference from May 16 to 18, 2004, is still in its preliminary phase.

5. Este articulo es un resumen de un trabajo de investigación hecho para la maestria en ciencias de la información en la Universidad de Montreal y dirigido por Marcel Lajeunesse.

6. El proyecto, presentado al Congreso de ACEAS (Asociación Francocanadiense para el Avance de las Ciencias) que tuvo lugar del 16 al 18 de mayo de 2004, se encuentra todavía en estado embrionario. void by studying the collection of rare books that once belonged to the Chevalier de Johnstone, which is, in a way, the heart and sole of the Library.

A systematic inventory of collections ${ }^{4}$ of rare books in Québec was recently undertaken by the Bibliothèque nationale du Québec (BNQ) and the Association québécoise pour l'étude de limprimé ( $A Q E E I$ ) and we hope that the inventory of this rich collection would highlight one of the many contributions made by David $M$. Stewart to the cultural fabric of his country.

Following a brief presentation of the man and his philosophy, we discuss the origins and the development of this special collection, which is, in fact, the reconstitution of a gentleman's library in $18^{\text {th }}$ century New France. A statistical analysis and a survey of the titles allow us to appreciate the extent to which David M. Stewart achieved his goal.

\section{Colección de libros antiguos del Caballero de Johnstone}

Mientras el Museo Stewart del Fuerte de la Isla Santa Helena se prepara para celebrar sus cincuenta años (1955-2005) rindiendo homenaje a su ilustre fundador, nos parece el momento oportuno para hacer un trabajo de investigación que ponga de relieve la biblioteca David M. Stewart. Esta biblioteca de museo, particularmente apreciada por el gran mecenas canadiense y a la que personalmente dedicó todos sus cuidados, nunca ha sido estudiada. Por lo tanto queremos llenar este vacio proponiendo el primer estudio exploratorio de la colección de libros antiguos del Caballero de Johnstone que constituye de cierto modo el alma misma de la biblioteca.

Por otra parte, dado que la Biblioteca Nacional de Quebec (BNQ) y la Asociación Quebequense para el Estudio del Material Impreso (AQÉI) iniciaron recientemente un proyecto de inventario sistematizado ${ }^{6}$ de las colecciones de libros antiguos que se encuentran en Quebec, esperamos que al preparar una descripción de esta rica colección, nuestra obra servirá para presentar a los investigadores este testimonio de una de las numerosas contribuciones de David M. Stewart al enriquecimiento cultural de su tierra.

Después de presentar brevemente el hombre y su filosofia, abordaremos el origen y el desarrollo de esta colección particular que quiere ser, en efecto, una reconstitución tipica de la biblioteca de un caballero de la Nueva Francia en el siglo XVIII. Un análisis estadistico y un vistazo de los títulos nos permitirán ver en qué medida David $M$. Stewart logró la misión que se proponía. 


\section{DAVID M.STEWART (1920-1984)}

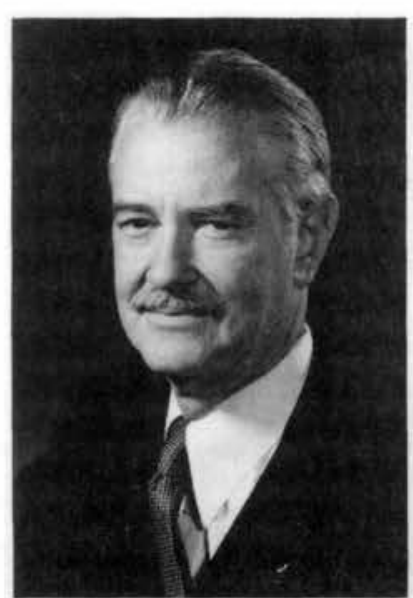

$\mathrm{H}$

éritier de la fortune acquise par sir William Macdonald (1831-1917), magnat de l'industrie canadienne du tabac, Walter Stewart, à son décès en 1968, transmet à son fils l'empire de la Macdonald Tobacco inc. Montréalais d'origine écossaise, David M. Stewart n'hérite pas seulement d'une fortune colossale, mais aussi d'une longue tradition de mécénat à laquelle il désire se vouer pleinement. Ainsi, en 1973, cinq ans à peine après avoir obtenu la direction de l'entreprise, il vend la compagnie à des intérêts américains et crée la Fondation Macdonald Stewart ${ }^{7}$. Voulant se consacrer entièrement à sa vocation philanthropique, il ne voyait pas de meilleure façon d'y parvenir ${ }^{8}:$ " [...] je gagne beaucoup plus que ce que j'aurais pu recevoir en m'occupant de la compagnie, en achetant de nouvelles machines, en investissant. Je voulais créer. M'intéresser à autre chose qu'aux affaires.” (Laforest, 1984 : 118).

La véritable passion de David M. Stewart, c'est l'histoire canadienne. Anglophone, il est un francophile avoué :

"Malgré que je sois canadien de langue anglaise, je dois affirmer que cette culture française me touche de près, c'est ma culture, mon héritage [...]. Car je suis né au Canada et je suis fier de ma patrie. Lépoque de Louis XIV m'est plus chère que celle de GeorgeIV ou Édouard VI d'Angleterre."

(Stewart, $1965: 6$ ).

À ses yeux, la Conquête de 1763 marque un tournant historique après lequel les deux cultures sont désormais appelées à vivre ensemble. Il revendique donc pour lui cet héritage de la Nouvelle-France et de ses grandes figures historiques, qu'il n'hésite pas à appeler "mes" héros. D'ailleurs, l'essentiel de sa conception de l'histoire canadienne est résumé sur un blason ${ }^{9}$ qui orne la porte de son premier musée en $1955^{10}$ : "Le Canada est l'héritier des deux grandes civilisations traditionnelles de l'ouest de l'Europe. Il lui appartient de les développer et il devrait en être fier." (Tari, $1991: 10$ ).

Fait pour l'action, David M. Stewart n'attend pas la création de sa fondation pour se mettre à l'œuvre. Vers le milieu des années 5o, alors qu'il déplore le laxisme des divers paliers de gouvernement dans la promotion et la protection de notre patrimoine national, il prend sur lui de donner l'exemple. Il crée ainsi, successivement, la Société historique du lac Saint-Louis (1954), le Musée militaire de lîle SainteHélène (1955), qui deviendra le Musée Stewart au Fort de lîle Sainte-Hélène, reconstitue deux régiments ayant combattu en Nouvelle-France ${ }^{11}$ (1962-1965), redonne vie au navire, grandeur nature, de Cartier (1967), contribue à la rénovation du château Ramezay (1970), puis inaugure un musée d'arts décoratifs au château Dufresne (1979). Enfin, à l'aube des années 80, il offre un dernier présent à la postérité en achetant et sauvegardant le manoir ${ }^{12}$ de Jacques Cartier, célèbre découvreur du Canada.

Généreux et altruiste, David M. Stewart souhaite, par ses efforts, communiquer sa passion de l'histoire. Son épouse le rappelait au cours d'une entrevue en 1996 : "David was not an individual collector. He never collected just for himself but to show other people» (Nowell, $1996: 23$ ). Le principal intéressé lui-même l'avouait humblement : " [...] tout ce que jai pu acquérir depuis 25 ans, cétait toujours dans le but de partager avec mes concitoyens et de les amener à s'intéresser à la vie quotidienne d'autrefois." (Brosseau, 1999 : A1).

Pour son apport majeur à la vie culturelle tant au Québec et au Canada qu'en Europe, il a été fait officier de l'Ordre du Canada et chevalier de la Légion d'honneur. Depuis son décès en 1984, son épouse, Liliane M. Stewart, qui a été de toutes ses entreprises, poursuit toujours avec la même vigueur l'œuvre commune.

\section{LA BIBLIOTHÈQUE DAVID M.STEWART}

Situé au sein du Musée Stewart au Fort de l'île Sainte-Hélène, le fonds de la bibliothèque rassemble deux collections de livres anciens dont la principale est celle du chevalier de Johnstone ${ }^{13}$. Couvrant la période des débuts de l'imprimerie jusqu'à la fin du Régime français en 1763, elle compte plus de 1200 titres. La seconde collection, intitulée John Graves Simcoe ${ }^{14}$, couvre le Régime anglais jusqu'à la Confédération, soit de 1764 à 1867 . Cette collection compte environ 200 titres. Une collection d'ouvrages de référence de

\footnotetext{
Fondation qui poursuit la longue tradition de mécénat héritée de Sir William MacDonald dans les domaines de léducation, de la santé et du patrimoine.

8. Il était aussi mal à l'aise avec le fait que le tabagisme avait de plus en plus mauvaise presse.

9. Ce blason est aujourd'hui bien en évidence sur un des murs de la bibliothèque.

10. Le Musée militaire de lìle Sainte-Hélène débute en 1955 dans un blockhaus situé sur liile.

11. La Compagnie franche de la Marine (1962) et le $78^{\text {th }}$ Fraser Highlanders (1965).

12. Manoir de Limoëlou, Saint-Malo, France. Aussi transformé en musée, en 1984.

13. Fait particulier, David M. Stewart a identifié ses propres collections de livres anciens aux noms de ses héros militaires. Il ne s'agit donc pas des collections personnelles du chevalier de Johnstone ou de John Graves Simcoe.

14. Célèbre militaire britannique qui s'est distingué pendant la Révolution américaine et qui fut lieutenant-gouverneur du Haut-Canada de 1791 à 1796.
} 
plus de 6500 titres complète les premières. Le fonds contient aussi plus de 600 cartes géographiques couvrant l'évolution de la cartographie du XVe siècle au XVIII ${ }^{e}$ siècle et où figurent des réalisations de tous les grands cartographes du temps, tels Bellin, Blaeu, Coronelli, DeBry, Delisle, Abbeville, Ruscelli, Sanson.... La collection d'estampes, composée de plus d'un millier de titres, gravés par les plus célèbres artistes de leur époque, couvre quant à elle presque tous les aspects de la vie sociale de l'Ancien Régime tant en Nouvelle-France qu'en Europe. Plus de 500 documents d'archives, imprimés ou manuscrits, sur lesquels figurent les signatures de personnages historiques comme Champlain, Frontenac, Lévis, Talon, Wolfe et Montcalm enrichissent le fonds. Enfin, des centaines de journaux d'époque, remontant aussi loin que 1611, complètent la collection.

\section{HISTORIQUE DE LA COLLECTION}

C'est en 1954 que l'idée de constituer une bibliothèque de livres anciens prend naissance. Cette annéelà, David M. Stewart met sur pied la Société historique du lac Saint-Louis afin de stimuler l'étude et la conservation du patrimoine historique québécois et canadien. Parmi les 13 objectifs que se fixe alors la Société se trouvent ceux de fonder un musée et de "créer une bibliothèque de livres historiques et précieux" (Procès-verbal, 1954:2) ${ }^{15}$. Premier titre de la collection, l'édition de 1632 des Voyages de Champlain devient aussi l'objet fondateur du Musée militaire de l'île Sainte-Hélène, qui voit le jour l'année suivante dans un petit bâtiment militaire de l'île. Dès lors, les chemins du Musée et de la bibliothèque seront inséparables alors que David M. Stewart met en place son concept de la valse de l'histoire : un livre, une estampe, un objet.

[Des livres rares] naquit l'intérêt pour les gravures qu'il se mit à collectionner. C'est alors que vint lidée d'agrémenter sa collection d'objets tridimensionnels. La joie de D.M.S. était alors de voir les livres accompagnés de vraies choses. Ainsi, le Musée offrait la particularité de présenter, et présente toujours, à la fois des livres, des gravures, des documents et des objets traitant d'un même sujet.

(Tari, $1991: 20$ ).

15. Il est intéressant de noter ici que les collections du Musée et de la bibliothèque appartiennent encore aujourd'hui à la dite Société.

16. Ne pouvant affronter en son âme et conscience ses compatriotes écossais qui combattent sous Wolfe, il se contente d'un rôle d'interprète. Il s'est aussi vu refuser une promotion.

17. Et pour la première fois un bibliothécaire professionnel est embauché afin de prendre soin de la collection.
David M.Stewart ne veut pas assembler n'importe quelle collection de livres anciens. Ce qu'il désire, c'est reconstituer une bibliothèque qui ferait l'envie d'un gentilhomme du XVIII ${ }^{\mathrm{e}}$ siècle établi en Nouvelle-France. Les Mémoires du chevalier de Johnstone, un de ses héros, sont à l'origine de cette inspiration romantique.

Militaire écossais à la solde de la France et aide de camp du général Montcalm pendant la guerre de Sept Ans, ce militaire frustré ${ }^{16}$ et souffrant d'ennui passe son temps libre à lire les quelques ouvrages qu'il a amenés avec lui :

Puysegur, Polybius, with the Commentaries of Folard, Feuguiere, Vegetius, the Commentaries of Caesar, Turenne, Montecuculi, Prince Eugene, Josephus, the Roman History, and Vauban, and other books of the same description, served me for killing the time, to dispel the evils of my position.

(Johnstone, $1870: 179$ )

La mention de ces quelques titres est l'étincelle qui enflamme l'imagination de David M. Stewart et le pousse à vouloir recréer la bibliothèque d'un officier gentilhomme de l'époque. Pour rendre hommage au héros qui l'a inspiré, David M. Stewart va désormais nommer sa collection «collection du chevalier de Johnstone».

Le Musée ayant alors une vocation militaire, c'est tout naturellement que les premières acquisitions de la bibliothèque vont porter sur l'art militaire. Cependant, ce concept d'une bibliothèque d' $d^{\prime}$ officier gentilhomme » va rapidement évoluer vers celui d'une bibliothèque d' «honnête homme» à mesure que le Musée prend de l'expansion et adopte une vision de plus en plus globale de l'histoire canadienne.

C'est à partir de 1973 que cette nouvelle orientation se fait clairement sentir. David M. Stewart ayant vendu son entreprise de tabac et créé la Fondation Macdonald Stewart, il est désormais libre de se consacrer entièrement au développement de sa bibliothèque et de son musée : les collections prennent alors leur envol ${ }^{17}$. D'un fonds d'environ 200 titres à cette époque, essentiellement sur l'art militaire, la collection de la bibliothèque s'agrandit rapidement pour couvrir l'ensemble du spectre de la connaissance au XVIII ${ }^{e}$ siècle. En 1976, la collection se trouvant déjà à l'étroit, le Musée entreprend un considérable projet de relocalisation de la bibliothèque qui durera trois ans. Le 21 février 1979, la nouvelle bibliothèque est inaugurée en grande pompe. Son ambiance feutrée, ses cuirs et ses cabinets de chêne, son système de climatisation contrôlé et son espace de rangement 


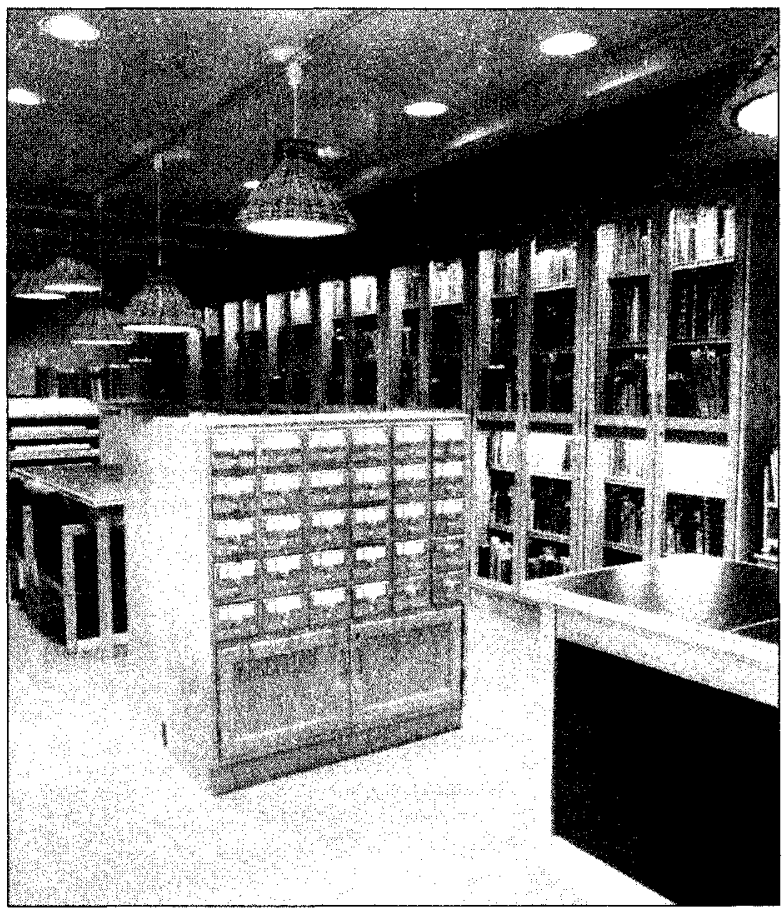

pour 10 ooo ouvrages en font désormais l'orgueil du Musée.

Curieusement, entre 1979 et 1983 , David M. Stewart songe un moment à ne plus identifier sa collection à son héros Johnstone. C'est qu'il a découvert une nouvelle source d'inspiration en la bibliothèque de Samuel Pepys au Magdalene College de Cambridge. Cette bibliothèque personnelle de 3 ooo titres réunis par Samuel Pepys ${ }^{18}$ (1651-1713) est particulière du fait que le nombre de titres en a été fixé dès le départ. Tout au long de sa vie, Pepys ne fera quaméliorer le contenu de sa collection. Impressionné, David M. Stewart songe alors à imiter Pepys et à fixer la taille de sa collection à 1 ooo titres : "I see now this collection being eventually as the Pepys Library, a closed collection of $x$ numbers of books» (Stewart, 1981: 1). Un an plus tard, en 1982, il paraît décidé : "[I think] we can now call it a 1 ooo rare Book Library, as we have now this number of volumes » (Stewart, $1982: 1$ ). En 1983 toutefois, alors que la collection dépasse les 1 ooo volumes, l'idée semble perdre progressivement de son attrait. Il hésite bien un instant à la qualifier de «over a thousand books collection», mais y renonce puisqu'elle est toujours de nos jours intitulée "collection du chevalier de Johnstone".

Une chose est sûre, cette collection demeure son principal intérêt jusqu'à son décès prématuré en 1984 et elle est bien aujourd'hui à l'image de ce qu'il souhaitait :

[What I want is] a library that has a soul and a character (and a heart)--and not simply a great mass of information with availability more important than the love given to such a collection of rare and precious objects.

(Stewatt, 1980:6)

\section{UNE BIBLIOTHÈQUE DU XVIIIE SIĖCLE}

Dans cette volonté de reconstituer une bibliothèque typique du milieu du XVIII ${ }^{e}$ siècle, quel degré d'authenticité David M. Stewart a-t-il cherché à atteindre? Réputé pour son intelligence et sa grande érudition, il apparait clairement, nous le verrons plus loin, qu'il s'est avant tout assuré d'une bonne représentativité des titres que l'on pouvait trouver à cette époque dans de telles bibliothèques. Il ne souhaite cependant pas reproduire de manière pointue la bibliothèque d'un individu ayant une profession ou un rang social donné. C'est donc à dessein qu'il la qualifie de bibliothèque d'un "honnête homme" afin de ne pas s'imposer de limites trop restrictives. Ainsi, s'il avait voulu reconstituer exactement la bibliothèque type d'un militaire en Nouvelle-France, celle-ci n'aurait pas été de grande envergure. Nous savons que Johnstone, son inspiration, ne possédait que quelques volumes. Le gouverneur Vaudreuil (1698-1778) n'avait lui aussi qu'une quinzaine de volumes et l'ingénieur Chaussegros de Léry (1682-1756), une cinquantaine. L'officier Joseph Fournerie de Vézon (1739-1760) avait la plus imposante collection pour un militaire avec 377 volumes (Drolet, $1965: 27-58$ ). On est donc loin des 1245 titres et des 1600 volumes qui composent aujourd'hui la collection.

Toutefois, si l'on examine les collections de l'intendant Claude-Thomas Dupuy (1686-1737), de l'entrepreneur François-Étienne Cugnet (1688-1751) et du conseiller du roi Louis-Guillaume Verrier (169o1758), hommes qui correspondent tous au qualificatif d' "érudit gentilhomme», la comparaison devient des plus intéressantes. Nous savons ainsi que la bibliothèque de l'intendant Dupuy contenait au minimum 6oo titres pour 1100 volumes (Dubé, 1965:222) et que celle de Cugnet était plus considérable avec plus de 8 oo titres pour 1600 volumes (Nish, 1975 147). Quant à celle de Verrier, le Dictionnaire biographique du Canada l'évalue à environ 1 ooo titres (dont plusieurs en de nombreux volumes), que Drolet chiffre à 2400 volumes (Drolet, $1965: 37$ ). Parmi ses critères d'authenticité, David M. Stewart n'a probablement pas vraiment considéré la taille de sa collection. Il est néanmoins intéressant de constater qu'elle s'avère tout de même réaliste pour une bibliothèque d'érudit en Nouvelle-France au XVIII ${ }^{\mathrm{e}}$ siècle. Ceci dit, il faut préciser qu'une étude de Daniel Mornet a montré que, sur 500 bibliothèques parisiennes du XVIII ${ }^{\mathrm{e}}$ siècle, 24 seulement avaient entre 600 et 700 titres (Dubé, 1969 : 333). Les bibliothèques de Dupuy, Cugnet et Verrier sont donc aussi quelque peu hors normes, surtout si l'on considère qu'elles ne sont pas à Paris, mais bien en Nouvelle-France ${ }^{19}$.

\footnotetext{
18. Secrétaire de l'Amirato britannique.

19. Ite missionnaire Navteres écrivait en 1737: "Lex lives somt aussi communs en France que rares en a pays (le Canada) oi it H'y a ni imprimeric ni librairie." (D)olet, 1965, p. 5 )
} 
En ce qui concerne le contenu, nous savons que les bibliothèques du XVIII ${ }^{e}$ siècle échappent généralement à la spécialisation et sont plutôt du type encyclopédique. De plus, «rares sont celles où les cinq classes traditionnelles (théologie, jurisprudence, sciences et arts, lettres, histoire) de la librairie ancienne ne sont pas représentées.» (Delon, 1997 : 159). S'il est vrai que ces cinq classes sont presque toujours présentes, elles le sont cependant dans des proportions si différentes d'une bibliothèque personnelle à l'autre qu'il serait hasardeux d'en généraliser quelque règle que ce soit. C'est que ces bibliothèques personnelles sont le plus souvent étroitement liées à l'occupation professionnelle du collectionneur ou à son domaine d'intérêt particulier. Encore là, la collection d'un individu peut s'avérer très différente de ce à quoi on pourrait s'attendre. Drolet cite ainsi le cas du militaire Simon Dupuis, qui compte 10 ouvrages religieux sur les 12 en sa possession, et du chirurgien Maheut, qui ne possède qu'un seul titre de médecine dans sa bibliothèque (Drolet, $1965: 39$ ). Autre exemple, les ouvrages religieux, qui sont très présents dans les collections d'avant 1760 , varient ainsi considérablement d'une collection à l'autre. Chez Dupuy, ils correspondent à $30 \%$ du total, chez Cugnet à $17 \%$, chez Vézon à $11 \%$ et chez Verrier à seulement $10 \%$.

Si la théologie, le droit, les lettres et l'histoire occupent une bonne place dans ces collections, on peut dire aussi que les bibliothèques personnelles de la Nouvelle-France se caractérisent par l'attention particulière qu'elles portent à certaines connaissances telles que la médecine, la navigation, l'astronomie, les sciences naturelles, l'agriculture, «toutes des sciences indispensables dans un pays où tout est $\grave{a}$ faire.» (Drolet, $1965: 57$ ). En somme, nous pouvons dire qu'en dehors de ces grandes lignes, il est presque impossible de dresser un portrait type parfait des bibliothèques personnelles du XVIII ${ }^{e}$ siècle, celles-ci étant, par nature, à l'image de leurs propriétaires.

\section{ANALYSE STATISTIQUE}

Bien que David M. Stewart ait développé son propre schéma de classification utilitaire, tout comme Dupuy et Cugnet, nous avons choisi de présenter les données selon le schéma classificatoire décimal de Dewey, qui est bien connu et qui dresse, à notre avis, un portrait synthétique plus juste du contenu de la collection. Voici d'abord la répartition des 1245 titres selon les 10 premières classes de Dewey :

\begin{tabular}{|c|c|c|}
\hline 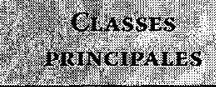 & $\begin{array}{l}\text { SOUS-CLASSE LA PLUS } \\
\text { IMPORTANTE }\end{array}$ & Titres \\
\hline $\begin{array}{l}\text { Géographie et } \\
\text { histoire }\end{array}$ & Géographie et voyages & 123 \\
\hline $\begin{array}{l}\text { Sciences } \\
\text { sociales }\end{array}$ & $\begin{array}{l}\text { Administration } \\
\text { publique (science } \\
\text { militaire) }\end{array}$ & 121 \\
\hline
\end{tabular}

\begin{tabular}{|c|c|c|}
\hline $\begin{array}{l}\text { Ciasses } \\
\text { PRINGIPAIES }\end{array}$ & $\begin{array}{c}\text { SOUS-CLASSE LA PLUS } \\
\text { IMPORTANTE }\end{array}$ & Titres \\
\hline $\begin{array}{l}\text { Sciences } \\
\text { appliquées }\end{array}$ & Sciences médicales & 60 \\
\hline Sciences pures & $\begin{array}{l}\text { Astronomie et sciences } \\
\text { connexes }\end{array}$ & 67 \\
\hline $\begin{array}{l}\text { Littérature et } \\
\text { rhétorique }\end{array}$ & $\begin{array}{l}\text { Littératures des langues } \\
\text { romanes }\end{array}$ & 36 \\
\hline Arts & $\begin{array}{l}\text { Loisirs et arts du } \\
\text { spectacle }\end{array}$ & 21 \\
\hline Religion & $\begin{array}{l}\text { Églises locales et ordres } \\
\text { religieux chrétiens }\end{array}$ & 19 \\
\hline Philosophie & $\begin{array}{l}\text { Phénomènes } \\
\text { paranormaux }\end{array}$ & 19 \\
\hline Langues & $\begin{array}{l}\text { Langues romanes. } \\
\text { Français }\end{array}$ & 6 \\
\hline Généralités & $\begin{array}{l}\text { Bibliothéconomie et } \\
\text { science de l'information }\end{array}$ & 6 \\
\hline
\end{tabular}

On observe aisément ici que la classe « Géographie et histoire " est dominante avec près du tiers des titres de la collection. Si les sciences sociales se trouvent au deuxième rang, c'est que cette catégorie contient la sous-classe "Science militaire", particulièrement bien représentée. Le Musée s'orientant de plus en plus vers les sciences depuis le début des années 80 , avec de riches collections de globes terrestres et célestes et d'objets scientifiques divers, les sciences arrivent immédiatement après l'histoire et l'art militaire. La littérature, les arts, la philosophie, la religion, les langues et les généralités se partagent le reste et ne comptent ensemble que pour $21 \%$ de la collection.

Un examen des subdivisions les plus importantes de chacune des classes principales permet une analyse plus fine du contenu.

Ce tableau particulièrement intéressant précise les points forts de la collection. Ainsi, l'on voit que dans la catégorie «Géographie et histoire» ce sont surtout les récits de voyages et les ouvrages reliés à la géographie qui occupent le haut du pavé. Dans les sciences sociales, pas de surprise, c'est l'art militaire qui occupe presque entièrement l'espace. La médecine occupe le premier rang dans la catégorie des sciences appliquées, alors que c'est l'astronomie dans celle des sciences pures. La sous-classe "Littérature" nous indique que les ouvrages de cette catégorie sont surtout de langue française. Dans celle des "Arts», ce sont surtout les loisirs et les arts du spectacle qui se démarquent. Du côté des ouvrages religieux, on trouve essentiellement ceux d'églises locales chrétiennes, c'est-à-dire, dans le cas qui nous occupe, ceux des diverses communautés religieuses ayant œuvré en Nouvelle-France. La sousclasse "Philosophie» ne contient pas les ouvrages des grands philosophes de l'Antiquité qu'on trouve traditionnellement, mais plutôt des ouvrages sur 
les phénomènes paranormaux comme l'occultisme, l'alchimie et la sorcellerie. Enfin, les dictionnaires de langue française sont mieux représentés et, dans la catégorie "Généralités", les ouvrages traitant des bibliothèques sont majoritaires.

Il faut toutefois descendre au niveau suivant des 100 premières classes de Dewey pour obtenir le portrait le plus précis possible. Dans la classe "Géographie et histoire", on constate que les récits de voyages sont suivis de près par les livres d'histoire de l'Europe et de l'Amérique du Nord et par la classe «Biographie, généalogie, emblèmes». Dans la catégorie des sciences appliquées, l'économie domes tique et la vie familiale suivent la médecine. Dans la catégorie des sciences pures, l'astronomie est suivie par les sciences naturelles, puis la physique et les mathématiques. Quant aux Belles-lettres, si la catégorie supérieure était majoritairement française, on constate que la littérature anglaise suit de très près. Enfin, la classe "Sciences sociales" montre qu'après la science militaire ce sont les «coutumes, moeurs et folklore» et le droit qui dominent. Un survol rapide des 100 premières classes de Dewey permet de voir que David $M$. Stewart a réussi à rassembler une collection qui couvre étonnamment bien les divers champs de la connaissance. En fait, la plupart des classes qui ne sont pas représentées dans la collection sont des disciplines qui n'existaient tout simplement pas au XVIII" siècle (photographie, médias d'information, paléontologie, etc.).

Examinons maintenant la dispersion chronologique de la collection:

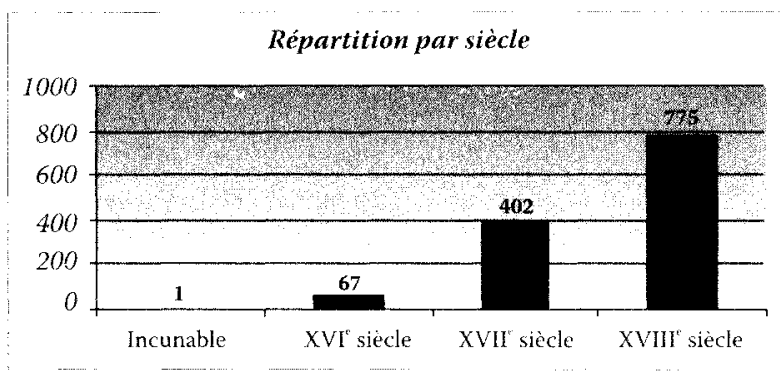

Cette figure nous indique que le XVIII ${ }^{\mathrm{e}}$ siècle est prédominant, avec près du double des titres du XVII ${ }^{\circ}$ siècle. Lécart est encore plus considérable comparé au XVIc siècle, qui en compte 10 fois moins. Sans tenir compte du pourcentage d'écart entre les siècles, cette répartition est tout de même conforme à la progression de l'édition, qui est constante depuis les débuts de l'imprimerie. L'édition française au XVIII siècle est en hausse (Chartier, 1982:118-123) et la collection est particulièrement riche en ouvrages de langue française. Ils correspondent, en fait, à $54 \%$ des titres du XVIII" siècle.

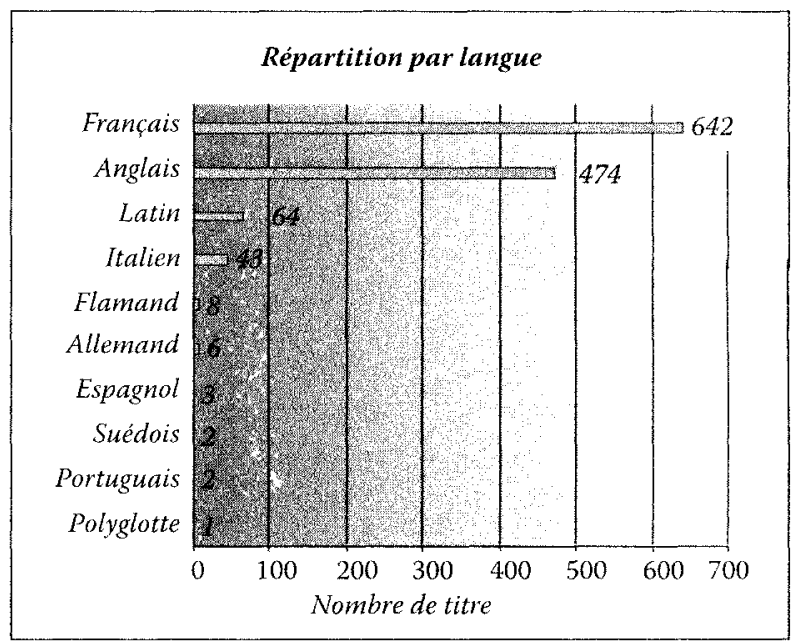

Comme on peut le constater, la collection est constituée principalement d'ouvrages en français et en anglais. C'est que David M. Stewart aimait s'imaginer que son «honnête homme » était un anglophone francophile. Il était donc important pour lui que cette bibliothèque soit bilingue. Aussi plusieurs des titres importants de la collection s'y trouvent-ils dans les deux langues.

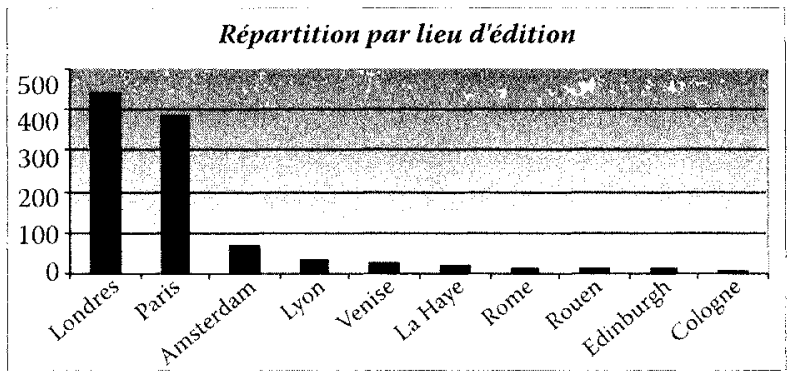

Cette figure ${ }^{20}$ indique les principaux lieux d'édition des ouvrages constituant la collection. Londres, Paris et Amsterdam, qui figurent depuis longtemps parmi les hauts lieux de l'édition, y sont les lieux de publication les mieux représentés. Bien que les ouvrages de langue française soient prédominants, la ville de Londres est au premier rang. Nous savons cependant que la contrefaçon est depuis longtemps florissante et que beaucoup d'ouvrages publiés en France portent souvent des «adresses vagues et fausses: Amsterdam, Genève et Londres" (Labarre, 1970 : 100).

Il faut aussi noter la grande variété d'auteurs qui composent cette collection avec près de 875 noms d'auteurs individuels ou collectifs pour une moyenne de 1,4 titre par auteur. Le plus représenté dans cette collection est sans aucun doute l'abbé Nollet, avec 11 titres, et Voltaire, qui vient immédiatement après avec 6 titres. Avec ces deux auteurs, on voit bien que cette collection n'est pas sans lien avec le Siècle des lumières, au cours duquel philosophes et scientifiques ont joué un rôle si important.

20. Lorthographe et la formulation des lieux de publication étant frop variées, nous nous contentons ici d'un ordre de grandeur. 
Jusqu'à présent, notre analyse nous permet de dire que la collection de David M. Stewart semble bien ajustée aux grandes lignes d'une bibliothèque du XVIII ${ }^{\mathrm{e}}$ siècle. Ainsi, la taille de la collection correspond en gros à la taille de celles que possèdent les érudits gentilhommes de la Nouvelle-France et son contenu est encyclopédique, avec une large place accordée à l'histoire et aux sciences. La théologie/religion et les Lettres classiques sont peut-être un peu sous-représentées, mais nous avons vu qu'il n'y a pas de règle absolue sur ce plan.

Encore plus révélatrice est cependant l'étude des titres que l'on trouve dans cette collection. Indépendamment des siècles, il existe toujours, dans les bibliothèques, un fonds commun d'ouvrages que l'on retrouve d'une collection à l'autre. Ainsi, une comparaison rapide des titres des collections de Dupuy, Cugnet, Verrier et Vézon avec ceux de la collection assemblée par David M. Stewart montre une correspondance certaine.

\section{LES TITRES DE LA COLLECTION}

David M.Stewart a sélectionné lui-même la quasi-totalité des titres de sa collection. À l'analyse, il apparaît qu'il a étudié attentivement la question et a fait des choix judicieux. Dans cette partie de notre étude, nous ne ferons qu'un survol des titres importants de la collection et qu'on trouve souvent dans les bibliothèques personnelles d'érudits au XVIII siècle.

Dans la classe des ouvrages militaires, qui fait en partie la réputation de cette collection, on trouve des titres qui vont des années 1532 à 1763 . C'est le magnifique et célèbre Re Military de Vegetius de 1532, entièrement illustré, qui ouvre cette classe. On y trouve aussi les Commentaires de César, dans une édition anglaise datant de 1655, et le très rare The New Highland Military Discipline, 1757 de Grant. En fait, la plupart des grands écrivains militaires s'y trouvent, de Folard ${ }^{21}$ - Nouvelles Découvertes sur la guerre dans une dissertation sur Polybe, 1724 - à Turpin de Crissé - Essai sur l'art de la guerre, 1724 - et Clairac - L'Ingénieur de campagne, ou traité de la fortification, 1749 -, en passant par Puységur - Art de la guerre, par principes et par règles, 1749 -, Leblond - Traité de l'artillerie, 1749 -, Belidor - Le Bombardier François, $1734^{22}$-, Alain $^{23}$ - Les Travaux de Mars, 1696 -, Baudoin - Exercice de l'infanterie françoise, 1757 - et Vauban

21. Folard, Puységur et César sont mentionnés, nous l'avons vu, par Johnstone dans ses Mémoires.

22. Lingénieur Chaussegros de Léry l'avait ici dans sa bibliothèque personnelle.

23. Présent dans la bibliothèque de Dupuy.

24. La réédition de la Société historique de Boucherville en 1664 n'en comptait que 5 dans le monde.

25. Fait particulier, une même gravure est souvent utilisée pour représenter des personnages différents ou des villes différentes.

26. Le rêve de David $M$. Stewart était de mettre la main sur une édition des relations de voyages de Cartier. Il n'aura malheureusement pu le réaliser.

27. L'évêque Lauberivière (1739-1740) le possède (Drolet, 1965: 51).
- Traité de l'attaque et de la défense des places, 174.2. Le très utile Traité général des subsistances militaires, 1744 de Dupré-d'Aulnay, indispensable à la compréhension du ravitaillement des troupes, est aussi présent dans la collection.

Dans la catégorie des ouvrages d'histoire, le bijou de la collection est sans aucun doute la première édition de 1613 des Voyages du sieur de Champlain, complet avec toutes ses cartes. La bibliothèque possède aussi l'édition de 1632, qui est le premier ouvrage acquis par David M. Stewart. Il faut aussi souligner cet exemplaire de l'Histoire véritable et naturelle des mours $\mathcal{E}$ productions du pays de la Nouvelle France, vulgairement dite le Canada, 1664 par Pierre Boucher. Cette œuvre de propagande, qui a contribué à faire de la Nouvelle-France une colonie royale, existe en moins de 10 exemplaires dans le monde ${ }^{24}$. Comme si ce n'était pas suffisant, cet exemplaire a appartenu à John Locke, célèbre philosophe anglais du XVII ${ }^{\mathrm{e}}$ siècle, et porte sa signature! La bibliothèque possède aussi l'Histoire de la Nouvelle France et Les Muses de la Nouvelle France, éditions de 1618, de Marc Lescarbot, ainsi que l'Histoire et Description générale de la Nouvelle France, 1744 de Charlevoix. Les Relations des Jésuites de Sagard, Le Jeune, Ragueneau, Vimont, Lallemant, Quen, Dablon et Rasle sont aussi présents.

La collection compte un seul incunable, mais pas n'importe lequel. Il s'agit de la deuxième édition, la plus recherchée, du Liber Cronicarum, 1497 de Schedel, qui est mieux connu sous le nom de Chronique de Nuremberg. Cette histoire du monde, en latin, avec caractères gothiques, compte plus de 1200 gravures sur bois ${ }^{25}$ et se trouve ici dans un état de conservation remarquable. Le Delle Navigationi et viaggi, 1556 de Ramusio est un des premiers livres d'histoire illustrés. Cette fameuse édition italienne contient les précieux récits de voyages de Jacques Cartier dont sa visite à Hochelaga ${ }^{26}$. Le Os Lvsiadas, 1613 de Camoes fait quant à lui le récit de l'explorateur Vasco de Gama. Le controversé Historie del S. D. Fernando Colombo, 1571 rédigé par le fils du grand explorateur de la mer Océane, Christophe Colomb, est une des sources les plus importantes concernant la vie du célèbre découvreur de l'Amérique. Le Historiae canadensis, 1664 de Pierre Cornut, que nous savons présent en NouvelleFrance ${ }^{27}$, est également sur les rayons.

On ne peut, bien sûr, mentionner ici tous les ouvrages d'histoire importants de la collection, mais soulignons que la bibliothèque possède, entre autres, et souvent en traduction, ceux de Bacqueville de la Potherie, Lahontan, Hennepin, Dièreville, Crespel, Lafiteau, Pouchot, Thévet, Bougainville, Kalm, Knox et Raleigh. Document particulier, une édition de 1727 de La Belle Assemblée a ses marges remplies d'un texte manuscrit d'un certain David Holden, militaire anglais qui relate sur 140 pages les derniers instants de la guerre de Sept Ans en Nouvelle-France. 
Les classes "géographie» et «exploration" rassemblent elles aussi de petits trésors. D'abord, L'Art de naviguer, 1554 de Pedro Medina, que l'on trouve dans de nombreuses collections personnelles en Nouvelle-France, et l'Histoire de la navigation... aux Indes Orientales, 1619 de Linschoten. Sont aussi présents les ouvrages de géographes de l'Antiquité : Pomponii Melae avec De sitv orbis, 1538 et Solinus Julius avec Polyhistor, 1538, ainsi que l'illustre $I l$ Theatro del mondo, 1598 d'Abraham Ortelius, première édition italienne du premier atlas connu. On y trouve la célèbre Cosmographei, 1561 de Sebastian Münster, l'Introduction à la géographie, 1743 du cartographe Nicolas Sanson et la superbe Description de l'Univers, 1683 d'Alain Manesson Mallet, en cinq volumes. Enfin, Richard Hakluyt nous présente The Principal Navigations, Voiages, Traffiques and Discoueries of the English nation, 1598, Samuel Purchas, son Pvrchas, his Pilgrimes in five bookes, 1628 et Olaus Magnus son Historia de gentibus septentrionalibus, 1555, le livre de géoethnologie le plus illustre de tout le $\mathrm{XVI}^{\mathrm{e}}$ siècle.

Dans la catégorie des Belles-lettres, le choix n'est pas négligeable. On y trouve six titres de Voltaire ainsi que les lettres de $\mathrm{M}^{\text {mes }}$ de Sévigny, de Maintenon et de Pompadour. Fait intéressant, la bibliothèque possède au moins trois titres aux armes de cette dernière et qui étaient dans sa bibliothèque personnelle. Un catalogue ${ }^{28}$ de sa collection, publié après son décès, nous confirme qu'il s'agit bien des ouvrages lui ayant appartenu. Rousseau, le mal-aimé, nous offre ses Euvres, 1756 et le premier opéra-comique français, $L e$ Devin du village, 1753 , qui a été présenté devant le roi à Fontainebleau en octobre 1752. CFuvres incontournables de la Renaissance, l'Éloge de la folie d'Érasme, version anglaise de 1709, avec les gravures d'Holbein, et l'Utopie ou Idée d'une république heureuse, 1730, de son ami Thomas Moore, ne manquent pas à l'appel, tout comme les Essais de Montaigne, en édition anglaise, 1693 et française, 1735. Le Décaméron, 1757 de Boccace, les Contes, 1699 de LaFontaine, et les Euvres, 1626 de Rabelais sont présents. Ainsi que des ouvrages de Corneille, Molière, Racine et Cyrano. Le fameux Don Quixotte de Cervantes, édition 1742, contient de magnifiques gravures. Du côté anglais, nous trouvons les Euvres, 1733 de Shakespeare, en sept volumes, Akenside, The pleasures of imagination, 1744 et The Life and Strange Surprizing Adventures of Robinson Crusoe, 1719 de Daniel Defoe. Des grandes figures de l'Antiquité, cependant, plusieurs manquent à l'appel, dont Salluste, Cicéron, Pline le Jeune et l'Ancien, Juvénal, Lucrèce, Suétone, Tacite, Térence et Sénèque. Nous trouvons néanmoins Catulle et Tibulle, Horace, Virgile et aussi le Works, $164 \mathrm{O}^{29}$, édition anglaise, du célèbre Flavius Josèphe. Du côté grec, il n'y a que l'Iliade, 1686 d'Homère, édition anglaise. Platon et Aristote ne sont pas présents. Terminons en soulignant l'incontournable Dictionnaire historique et critique, 1720 de Pierre Bayle et la présence de l'œuvre maitresse du Siècle des lumières, l'Encyclopédie, 1751-1780 de Diderot et d'Alembert, complète en 35 volumes.

La section des sciences est particulièrement intéressante. Dans le domaine de la médecine, tous les grands noms sont présents: Ambroise Paré ${ }^{\circ}$, premier chirurgien militaire du roi François $\mathrm{I}^{\mathrm{er}}$, avec ses Fuvres, 1607 et en version anglaise, 1649 ; William Harvey, médecin et physiologiste anglais célèbre pour ses découvertes sur la circulation du sang, avec The Anatomical Exercises concerning the motion of the heart and blood, 1673 ; André Vésale, médecin flamand père de l'anatomie moderne, avec Anatomia, 1604.

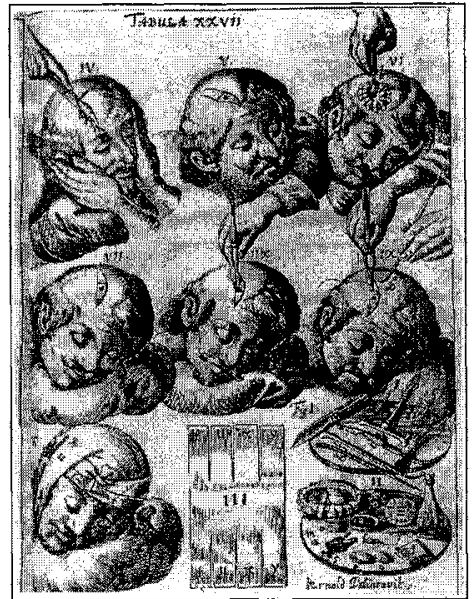

Dans son traité de chirurgie The Noble Experyence of the vertuous handy warke of surgeri, 1525 , première édition anglaise, le médecin allemand Hieronymus Brunschwig est le premier à parler du traitement des blessures par balles. Les gravures sur bois qui composent l'ouvrage sont parmi les premiers spécimens d'illustration de médecine.

Il y a aussi Johannes Scultetus avec L'Arcenal de chirvrgie, 1672, complètement illustré, et le classique Regimen Sanitatis Salerni : or, The Schoole of Salernes Regiment of Health, 1634. Ce best-seller, édité à plus de 300 reprises, traite de tous les aspects de la santé. Le Cours d'opérations de chirurgie, 1740 de La Faye présente le cours de chirurgie de M.Dionis, premier chirurgien de $\mathrm{M}^{\text {mes }}$ les dauphines. Il est intéressant parce que nous savons qu'il a servi de manuel à ceux qui suivaient le cours de $M$. Dionis, comme une note manuscrite de la page de garde semble l'indiquer : «Je suis le cours des opérations de chirurgie de Monsieur Dionis et j’appartiens à François Simon. Présent à la Charité des moulins, ce 12 juillet 1746.» Le Dictionnaire ou traité universel des drogues simples, 1727 de Nicolas Lémery, le «magicien de la rue Galande ${ }^{31} »$, est aussi présent dans la collection.

Un bijou, la première édition latine du Dialogus Systemate Mundi, 1635 du célèbre Galilée orne les rayons. Cet ouvrage, publié 17 ans après la condamnation du système copernicien, a tout de même été mis à l'index jusqu'en 1822. Il présente un dialogue

\footnotetext{
28. Remarquable au surplus car il est annoté de l'époque avec la valeur des ouvrages en marge.

29. Aussi mentionné par Johnstone.

30. Souvent présent dans les bibliothèques personnelles en Nouvelle-France.

31. En 1670 , Lémery ouvre un cours de chimie dans une cave de la rue Lalande à Paris. Le succès sera foudroyant et lui vaudra ce surnom.
} 
entre trois amis qui discutent des différences entre les conceptions copernicienne et ptoléméenne du monde. On y trouve aussi Clavdii Ptolemaei Alexandrini geographicae enarrationis, 1535 de Claude Ptolémée, édité par l'hérétique Michel Servet, qui périra sur le bûcher en 1553. La collection contient trois titres du non moins réputé Tycho Brahe, l'astronome au «nez d'or ${ }^{32}$ ". Il y a aussi la Sfera del mondo, 1564 de l'astronome Alessandro Piccolomini, le Qvadrans Apiani. Astronomicvs et iam recens inventvs, 1532 de Petrus Apianus, la Sphère dv monde, proprement ditte Cosmographie, 1552 de Finé Oronce, le Harmonia macrocosmica seu atlas universalis et novus, 1728 d'Andreas Cellarius et L'usage des globes céleste et terrestre et des sphères, 1728 de Nicolas Bion. La bibliothèque possède également des ouvrages de Coronelli, Willem Janszoon Blaeu et De Maupertuis.

Le grand Newton nous offre son Philosophiae Naturalis Principia mathematica, 1726 et René Descartes, le père de la philosophie moderne, ses Principes de la Philosophie, édition 1668 et en latin, 1664. L'abbé Nollet, éminent vulgarisateur des sciences, est présent dans la collection avec pas moins de 11 titres. Plusieurs d'entre eux, dont Nollet, Lettres sur l'electricité, 1753 et Franklin, Expériences et Observations sur l'électricité, 1753, illustrent le débat qui a opposé les deux quant à l'origine de l'électricité.

Dans le secteur de la religion, classe modeste, nous avons tout de même des titres très importants, parmi lesquels toute une série de Relations écrites par les missionnaires en Nouvelle-France, dont celles de Paul Le Jeune, de Jérôme Lallemant et de Barthelemy Vimont. La collection possède aussi le Rituel du diocèse de Québec publié par lordre de Monseigneur de Saint-Vallier évêque de Québec, 1703, un Missel de Paris, 1739, un Manuel des inquisiteurs, à l'usage des inquisitions d'Espagne $\mathcal{E}$ de Portugal, 1762 et une Vie de la mère Catherine de Saint-Augustin, religieuse hospitalière de la Miséricorde de Québec en la Nouvelle-France, 1671. Il y a, enfin, l'Histoire des diables de Loudun, 1752, qui «dénonce les cruels effets de la vengeance du cardinal Richelieu» et la mythique Histoire de la papesse Jeanne, 1694, deux ouvrages que possède Guillaume Verrier en Nouvelle-France.

Terminons par quelques titres amusants et/ou curieux. Il y a d'abord l'inattendu Le Moyen de devenir riche, 1636, du célèbre céramiste Bernard Palissy, les chicanes entre hommes et femmes avec Controuerses des sexes mascvlin et foemenin, 1541 de Gratien Dupont et De l'excellence des hommes contre l'égalité des sexes, 1675 de Labarre. On trouve aussi ce poème manuscrit unique et magnifiquement illustré de broderies intitulé Poëme sur sainte Élisabeth, présenté à Madame de Richelieu, abbesse de Saint-Remy, au jour de sa feste de sainte-élisabeth, le vingtième de Novembre de l'année 1705 et deux volumes du Diurnale Noviomense, 1774, dont les tranches de gouttière sont peintes, l'une d'une vue de Montréal et l'autre d'une vue de Québec. Ce qui fait la particularité de ces œuvres, c'est qu'elles ne sont visibles que lorsque la tranche est placée à 45 degrés. Il y a aussi la satire Histoire des rats pour servir à l'histoire universelle, $1737 ;$ L'Homme dans la lune: ou le voyage chimérique fait au monde de la lune, 1671; La Dernière Guerre des bêtes, fable pour servir à l'histoire du XVIII siècle, 1758 ; Les Hommes volans, 1763 ; et le comique The Art of ingeniously tormenting yourself, your attendants, and all your friends, 1757 . Enfin, la bibliothèque possède plusieurs éditions de l'illustre astrologue Michel de Nostre-Dame, dit Nostradamus.

La bibliothèque contenant plus de 1200 titres, nous pourrions continuer longtemps à énumérer des ouvrages intéressants. Nous en avons d'ailleurs sûrement oublié quelques-uns. Si, dans la nomenclature qui précède, tous ne se trouvent pas dans les bibliothèques personnelles en Nouvelle-France, nous en avons cependant repéré un grand nombre qui s'y trouvent. Une chose est sûre, à la lumière de ce que nous venons de voir, on peut assurément dire que la "collection du chevalier de Johnstone» est d'une grande richesse et que, sans nul doute, elle aurait fait l'envie de n'importe quel «honnête homme» ou «homme de goût» du XVIII ${ }^{\mathrm{e}}$ siècle.

\section{CONCLUSION}

La collection de livres anciens de la bibliothèque David M. Stewart est née d'une passion pour l'histoire et d'une inspiration littéraire opportune. Elle a aussi été au cœur de la naissance du Musée Stewart au Fort de l'île Sainte-Hélène, qui contribue depuis 50 ans à l'enrichissement culturel du Québec. Musée au sein d'un musée, cette bibliothèque a toujours été au cœur des intérêts de David M. Stewart. Il lui a accordé tous ses soins et toute son attention. Cette collection, il l'a assemblée dans le but de l'offrir aux futures générations et, signe de son altruisme, il n'a pas osé lui donner son nom. Si elle est d'une grande richesse, c'est qu'il a soigneusement choisi les titres qui allaient la composer. Le philosophe latin Sénèque disait d'une bibliothèque que ce n'était pas la quantité, mais la qualité qui importait. David M. Stewart aurait été entièrement d'accord avec lui. Notre étude a montré, nous l'espérons, que ce grand philanthrope canadien a su recréer avec succès une bibliothèque qui aurait fait l'envie de bien des lettrés du XVIII ${ }^{\mathrm{e}}$ siècle. Cette collection, David M. Stewart la voulait accessible. C'est pourquoi nous souhaitons que notre travail puisse modestement contribuer à la faire mieux connaitre.

32. Réputé pour son mauvais caractère, Brahe perd le bout de son nez dans un duel à l'épée. Il portera une prothèse en or toute sa vie. 


\section{Remerciements}

À Sylvie Dauphin pour ses commentaires toujours pertinents et pour son soutien. À $\mathrm{M}^{\text {mes }}$ Elizabeth Hale et Eileen Meillon, bibliothécaires de la bibliothèque David M. Stewart. A Marcel Lajeunesse et Gilles Deschâtelets pour l'occasion.

\section{SOURCES CONSULTÉES}

Bolton, Bruce D. Le Bibliothécaire de musée. Communication donnée à l'AMC. 1980, $9 \mathrm{p}$.

Boucher, Pierre. Histoire véritable et naturelle..., Boucherville, Société historique de Boucherville, 1964, $415 \mathrm{p}$.

Brosseau, Cécile. «La bibliothèque MacDonald Stewart au Musée militaire et maritime ». In La Presse, 27 février 1979.

Chartier, Roger et Martin, Henri-Jean. Histoire de lédition française : le livre triomphant, 1660-183o, tome 2, Paris, Promodis, 1982.

Delon, Michel (sous la direction de). Dictionnaire européen des Lumières, Paris, PUF, 1997, 1128 p.

Drolet, Antonio. Les Bibliothèques canadiennes 1604-1960, Canada, Le Cercle de France, 1965, 234 p.

Dubé, Jean-Claude. Claude-Thomas Dupuy, intendant de la Nouvelle-France, 1678-1738, Montréal, Fides, 1969, 395 p.

Fauteux, Ægidius. «Les bibliothèques canadiennes et leur histoire». Extrait de la Revue canadienne. Montréal, Arbour et Dupont, 1916, p. 97-114.
Furet, F, et al. Livre et Société dans la France du XVIII siècle, Paris, Mouton et cie, 1965-1970, 2 volumes.

Johnstone, chevalier de. Memoirs. Aberdeen, D. Wyllie \& Son, 187o, 3 volumes.

Labarre, Albert. L'Histoire du livre, Paris, PUF, 1979, coll. «Que saisje?»,126 p.

Laforest, Pierre. «Le plus généreux mécène du monde adopte Saint-Malo». Lactualité, 25 février 1984, p.114-121.

Nish, James Cameron. François-Étienne Cugnet, 1719-1751 : entrepreneur et entreprises en Nouvelle-France, Montréal, Fides, 1973, $185 \mathrm{p}$.

Nowell, Iris. "Liliane Stewart». In Nowell. Women who give away millions : portrait of Canadian philanthropists, Toronto, Hounslow Press, 1996, 40 p.

Procès-verbal. Société historique du lac Saint-Louis. Aim of the Society, 9 décembre 1954, $6 \mathrm{p}$.

Tari, Katy. David M. Stewart et le Musée militaire de lîle SainteHélène, Montréal, Université de Montréal, Mémoire de maîtrise en muséologie, 1991, 28 p.

Stewart. Pélican Pick, Montréal, 1965, 6 p.

Stewart ${ }^{1}$. Philosophy of the MS Library, Pointe-Claire, 27 avril 1980, $6 \mathrm{p}$.

Stewart ${ }^{2}$. Philosophy of the collections, Virginia Beach, 11 juillet 1981, $5 \mathrm{p}$.

Stewart ${ }^{5}$. The Philosophy \& Policy of the David M. Stewart Library, Londres, 8 juin 1982, $4 \mathrm{p}$.

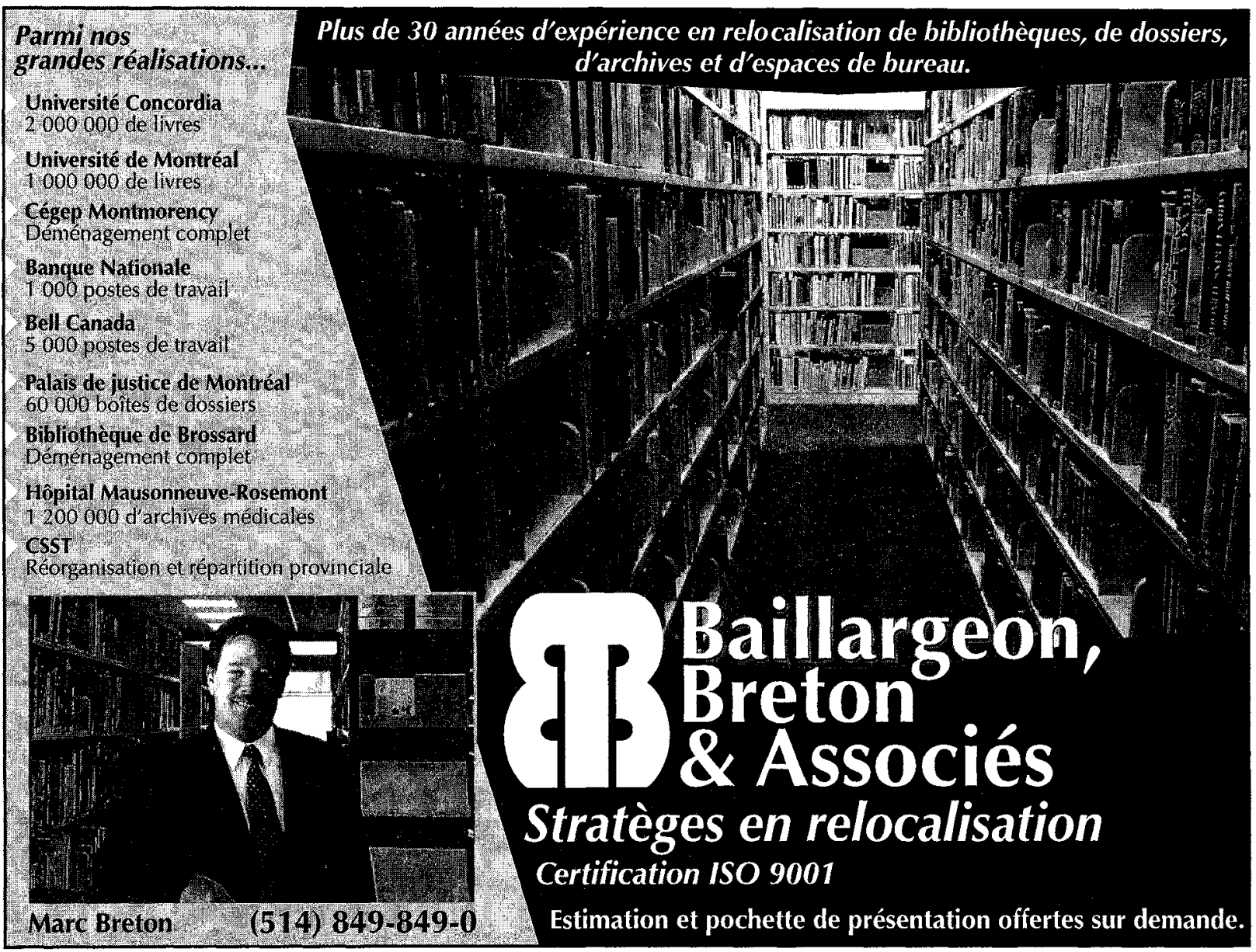

